Am J Hosp Palliat Care. 2017 November ; 34(9): 860-868. doi:10.1177/1049909116659049.

\title{
Are Trends in Hospitalization Prior to Hospice Use Associated With Hospice Episode Characteristics?
}

\author{
Brystana G. Kaufman, MSPH ${ }^{1}$, Carla A. Sueta, MD, PhD $^{2}$, Cathy Chen, BSE ${ }^{3}$, B. Gwen \\ Windham, MD, MHS $^{3}$, and Sally C. Stearns, $\mathbf{P h D}^{1}$ \\ ${ }^{1}$ Department of Health Policy and Management, The University of North Carolina at Chapel Hill, \\ Chapel Hill, NC, USA \\ 2Division of Cardiology, The University of North Carolina at Chapel Hill, Chapel Hill, NC, USA \\ ${ }^{3}$ University of Mississippi Medical Center, Jackson, MS, USA
}

\section{Abstract}

This study expands current knowledge of factors associated with initiation of hospice care by examining prehospice patterns of medical care leading to Medicare hospice use and the relationships to hospice episode characteristics. Data from the Atherosclerosis Risk in Communities (ARIC) study cohort offer the ability to control for measures that are not available in Medicare claims data, including marital status, nursing home residency, and education. For 1248 ARIC participants who used hospice (2006-2012), participant level trends in the number of hospital days per 30-day period over the year prior to hospice initiation were generated using a fixed-effects model. Logistic regression was used to estimate the associations between increasing hospital use over the year prior to hospice enrollment with key patient characteristics (diagnosis, age, and comorbidity) and episode characteristics (short hospice stay ending in death, long hospice stay, and live discharge). Participants with severe comorbidity (measured as a Charlson comorbidity index score greater than 5) had higher odds of increasing hospital use prior to hospice (odds ratio $[\mathrm{OR}]=3.28$, confidence interval $[\mathrm{CI}]=2.25-4.78$ ). Increasing hospital use did not vary by diagnosis but was associated with reduced odds of a live hospice discharge $(\mathrm{OR}=0.55$, CI $=0.34-0.88)$ or long stay in hospice $(\mathrm{OR}=0.44, \mathrm{CI}=0.24-0.79)$ and increased odds of a short stay in hospice $(\mathrm{OR}=1.92, \mathrm{CI}=1.36-2.71)$. The evidence that care patterns prior to hospice use are associated with hospice outcomes could facilitate development of interventions to improve timely hospice referral.

\section{Keywords}

hospice; palliative care; end of life; hospice episode length; hospice live discharge; Medicare

\footnotetext{
Reprints and permission: sagepub.com/journalsPermissions.nav

Corresponding Author: Brystana G. Kaufman, MSPH, UNC Gillings School of Global Public Health, 135 Dauer Drive, Campus Box 7411, Chapel Hill, NC 27599, USA. bkaufman@ live.unc.edu.

Declaration of Conflicting Interests

The author(s) declared no potential conflicts of interest with respect to the research, authorship, and/or publication of this article.
} 


\section{Introduction}

Hospice use improves the quality of end-of-life care.- In 2011, the National Hospice and Palliative Care Association estimated almost half (44.6\%) of all deaths in the United States were under the care of hospice; however, about one-third of hospice beneficiaries did not enroll until their last week of life.' Although a short hospice episode is not necessarily an indication of delayed use, many families perceive the timing of hospice enrollment as late, and this perception is more common with stays of less than 4 weeks.' The perception of delayed hospice enrollment is associated with more unmet needs, higher reported concerns, and lower satisfaction. Factors associated with hospice episode length or live discharge may inform strategies for timely initiation of hospice care, improving patient satisfaction and quality of life.

Health service use preceding hospice is associated with hospice episode length. Of beneficiaries enrolling in hospice in the last 3 days of life, $40 \%$ of episodes are preceded by hospitalization with intensive care days." After controlling for patient characteristics, hospice patients with the hospital or emergency department as their preadmission setting are more likely to experience a hospice stay of less than 1 week compared to those entering hospice from outpatient or long-term care settings. Diagnosis is a predictor of both patterns of care and hospice episode characteristics; cardiovascular disease (CVD) is associated with short stays in hospice, while patients with dementia are more likely to have long stays. ${ }^{-}$In addition, patients with heart failure are more frequently referred to hospice from hospitals and nursing facilities compared to patients with advanced cancer.

Although health services utilization preceding hospice is associated with hospice episode length, this relationship has not been explored in the context of care trajectories. This study uses data from a longitudinal cohort study to describe the intensity of hospitalization over the year before the initial hospice episode and examines the association of those trends with patient and hospice episode characteristics (length of episode and live discharge). An examination of prehospice patterns of hospitalization and association of these patterns with hospice episode length or live discharge may provide insights for improving the timeliness of hospice referral.

In our conceptual framework, intensity of care over the year prior to hospice is a determinant of hospice episode length, which impacts outcomes of care, including patient and care-giver satisfaction with care. Among possible measures of care intensity (eg, cost, emergency department visits, or intensive care unit use), hospital days are an important indicator because of their association with lower quality of life and higher mortality. -, Hospitalizations expose frail elderly to risks of nosocomial infections, delirium, pressure ulcers, falls, functional decline, and other adverse events. We hypothesize 2 relationships while controlling for other observed characteristics: (1) trends in hospitalization will differ by primary hospice diagnosis (eg, cancer, dementia, CVD, chronic obstructive pulmonary disease [COPD], or other conditions), comorbidity, and age; and (2) hospice episode characteristics including length of hospice episode (short vs long) and live discharge will differ by the trend in hospitalization prior to hospice initiation. 


\section{Methods}

\section{Data}

This retrospective study utilized longitudinal cohort data from the Atherosclerosis Risk in Communities (ARIC) study.' In 1987 to 1989, the ARIC study began follow-up of 15792 participants aged 45 to 64 years, selected by probability sampling from 4 communities: Forsyth, North Carolina; Minneapolis, Minnesota; Jackson, Mississippi; and Washington County, Maryland. Institutional review boards provided approval. Data sources included ARIC baseline data (ARIC center, race, education level, birthdate, and gender); annual follow-up (AFU) telephone interview (marital and nursing home status); data from the Centers for Medicare \& Medicaid Services (CMS) including enrollment, claims, Chronic Conditions Warehouse, and Medicare Provider and Analysis Review inpatient records; and information on hospitalizations from ARIC hospital surveillance (including hospitalizations for Medicare advantage enrollees or veterans).

This analysis identified hospice users using 2006 to 2012 Medicare hospice claims retaining only the first hospice episode in the period for each participant. Nonblack and nonwhite race groups were excluded due to small numbers $(\mathrm{n}=3)$ for a final sample of 1248. A subgroup analysis using 791 Medicare fee-for-service (FFS) enrollees included additional claims information on nursing home use and chronic conditions at the time of hospice initiation. We analyzed only Medicare hospice services due to lack of data on hospice services provided by Medicaid, the Veteran's Administration, or private sources.

\section{Prehospice and Hospice Episode Outcome Measures}

Intensity of care was measured using days hospitalized over the 360 days prior to hospice admission; days hospitalized (including observation stay days) were allocated into 12 periods of 30 days each, resulting in 12 observations per participant for the trend analysis. Short hospice episode was defined as an episode of 7 days or less prior to death; long hospice episode was defined as $>180$ days.' A dichotomous indicator of a live discharge from hospice was constructed.

\section{Covariates}

Mutually exclusive diagnosis categories were assigned by the primary or secondary International Classification of Diseases, Ninth Revision (ICD-9) diagnosis codes on hospice claims including dementia (331-335, 290-294, 797), CVD (390-439), cancer (140-239, $258,795-796,511.81,789.51)$, COPD (490-496), and other. The secondary ICD-9 code was only used for those initially assigned to "other" by the primary code. Additional patient covariates included gender ( 1 if female, 0 otherwise), age (65-74, 75-84, 85, or older), race (black or white), marital status ( 1 if married, 0 otherwise), education level (less than high school vs high school equivalent degree or higher), nursing home status, and comorbidity. ${ }^{-}$ Nursing home residency was defined by self- or proxy report during the AFU prior to hospice enrollment. The Charlson comorbidity index (CCI) was generated using software from the Agency for Healthcare Research and Quality to analyze hospitalization claims (Medicare and non-Medicare) from 2005 to 2012; weights for constructing the score were applied from the CCI. The CCI scores greater than 5 defined severe comorbidity. 
The FFS beneficiaries were identified as those with enrollment in Medicare part A and part B but no Medicare advantage enrollment at the time of hospice entry $(\mathrm{N}=794)$. The FFS subsample analysis utilized different measures of comorbidity and nursing home status. For this subsample, instead of using the CCI, the comorbidity score was defined as a binary indicator of more than 5 chronic conditions ever indicated in the Chronic Conditions Warehouse (1999-2013) within 6 months of hospice admission. The FFS nursing home admissions (skilled and long term) less than 60 days prior to hospice admission were identified using procedure and place of service codes from carrier claims, following a validated process with a sensitivity of $96.7 \%$.

\section{Statistical Design and Analysis}

Patient-level trends were defined by regressing the number of hospital days on person and time fixed effects plus their interaction, with the constant restricted to 0. Participants with no hospitalizations in the year prior to hospice were not included in the trend analysis $(\mathrm{n}=290)$. A binary indicator for a significant $(P<.05)$ and positive trend was included in subsequent models as the key variable of interest.

Five logistic regression models were used to predict 5 outcomes: any hospitalizations in the study period, a significant and positive trend in hospital use given any hospitalization, short hospice stay, a long hospice stay, and live discharge from hospice. All models controlled for patient characteristics (diagnosis, age, marital status, nursing home status, site/race, and comorbidity) and the available combinations of race and center, though racial diversity was present in only 1 of the 4 ARIC centers (Forsyth County, North Carolina). Participants with no comorbidity data due to no hospital visits $(\mathrm{N}=97)$ or only observation stays $(\mathrm{N}=3)$ in the year prior to hospice initiation were assigned a CCI score of 0 , and an indicator for missing comorbidity was added to the model ( 1 if missing, 0 otherwise).

\section{Results}

Table 1 provides descriptive statistics for the sample in total, subsample of FFS beneficiaries, and by diagnosis group. Of 1248 hospice users, the primary or secondary qualifying hospice diagnosis was listed as cancer in 40\%, CVD in 20\%, dementia in $13 \%$, and COPD in $6 \%$. The $21 \%$ other hospice qualifying diagnoses included indications of diseases of the respiratory and genitourinary systems as well as nutritional and metabolic disorders including frailty, failure to thrive, weight loss, and diabetes. About two-thirds of the sample had severe comorbidity (Charlson score of greater than 5) with a mean Charlson score of 6.37 in the sample. Using the Chronic Conditions Warehouse measure, 59\% of the FFS subsample had more than 5 conditions.

Overall, $25 \%$ of the participants initiated hospice within 7 days of death, whereas $15 \%$ experienced hospice stays longer than 180 days (Table 1). The median and mean initial hospice episodes were 26 and 85 days, respectively. Live discharges occurred in $23 \%$ of hospice episodes; this percentage reached $42 \%$ in the CVD diagnosis group. About half of hospice episodes for the sample occurred in freestanding facilities, whereas 1 in 3 occurred in for-profit facilities. 


\section{Patterns of Care Prior to Hospice}

In the year before hospice, $23 \%$ of hospice users had no hospitalization; this proportion was highest in the dementia diagnosis group at 35\% (Table 1). In the COPD diagnosis group, $25 \%$ of participants had a trend of increasing hospital use, compared to $20 \%$ in the CVD group, $18 \%$ in the other and cancer groups, and $10 \%$ in the dementia group. For participants with a hospitalization in the period, 3 patterns of hospital use by 961 participants emerged (Figure 1). Over the year before hospice, 220 participants had positive (increasing) trends in hospital use, whereas 741 had nonsignificant $(n=732)$ or negative trends $(n=9)$. Among those with significantly positive trends, hospital use increased at a rate of 0.5 to 2.4 inpatient days per 30-day period, with larger increases occurring over the 6 months prior to hospice. All 3 groups experienced an average increase in hospital use during the month prior to hospice.

\section{Full Sample Analysis}

Table 2 provides results from the 2 regressions assessing factors associated with any hospital use and significantly increasing hospital use during the study period. Factors significantly $(P$ $<0.05$ ) positively associated with any hospital admission (including observation stays) were a qualifying diagnosis of COPD relative to dementia (odds ratio $[\mathrm{OR}]=3.06$, confidence interval $[\mathrm{CI}]=1.37-6.87$ ), severe comorbidity (as measured by the CCI; OR $=6.20, \mathrm{CI}=$ 4.41-8.71), and high school education ( $\mathrm{OR}=1.48, \mathrm{CI}=1.07-2.05)$. For hospice users with at least 1 hospital admission in the study period, severe comorbidity was associated with increasing hospital use $(\mathrm{OR}=3.28, \mathrm{CI}=2.25-4.78)$. Age-group was also significant, with age older than 85 years associated with reduced odds relative to those under 75 years $(\mathrm{OR}=$ $0.46, \mathrm{CI}=0.26-0.83)$. The FFS status was associated with reduced odds of increasing hospital use $(\mathrm{OR}=0.64, \mathrm{CI}=0.45-0.92)$.

Table 3 shows results from the regressions assessing factors associated with hospice episode outcomes. Any hospital admission and increasing hospital use were associated with increased odds of a short hospice stay $(\mathrm{OR}=2.90, \mathrm{CI}=1.72-4.89$ and $\mathrm{OR}=1.92, \mathrm{CI}=$ 1.36-2.71, respectively). A hospice qualifying diagnosis of CVD or other diagnosis increased the odds of a short stay relative to dementia $(\mathrm{OR}=1.92, \mathrm{CI}=1.10-3.34$ and $\mathrm{OR}=$ $2.50, \mathrm{CI}=1.47-4.23$, respectively). Among patient characteristics, the odds of a short stay were reduced for those who were married $(\mathrm{OR}=0.65, \mathrm{CI}=0.46-0.93)$ or in a nursing home $(\mathrm{OR}=0.36, \mathrm{CI}=0.13-0.99)$.

Any hospital admission and increasing hospital use were associated with reduced odds of a long hospice stay $(\mathrm{OR}=0.39, \mathrm{CI}=0.25-0.59$ and $\mathrm{OR}=0.44, \mathrm{CI}=0.24-0.79$, respectively), as was a diagnosis of CVD or cancer relative to dementia $(\mathrm{OR}=0.45, \mathrm{CI}=0.25-0.80$ and $\mathrm{OR}=0.38, \mathrm{CI}=0.22-0.63$, respectively). Married participants had increased odds of long hospice stay $(\mathrm{OR}=2.04, \mathrm{CI}=1.39-3.01)$. Persons in a nursing home at the last telephone interview prior to hospice initiation had odds of a long hospice stay 2.5 times higher than nonnursing home residents $(\mathrm{OR}=2.54, \mathrm{CI}=1.14-5.68)$. Similarly for live discharge, odds were significantly decreased for those with a hospital admission or increasing hospital use $(\mathrm{OR}=0.33, \mathrm{CI}=0.21-0.52$ and $\mathrm{OR}=0.55, \mathrm{CI}=0.35-0.87$, respectively $)$. For those with 
cancer, older than 85 years, or missing comorbidity score, odds of a live discharge were reduced by more than half.

Site and race were also significantly associated with length of hospice episode and discharge status; for example, compared to Washington County participants (all white), Jackson participants (all black) had significantly increased odds of a long hospice stay and live discharge but reduced odds of a short stay. Correlation with location (eg, a limited number of hospice providers in the area) may have obscured the effect of hospice provider characteristics (profit status and freestanding status), which were not significant.

\section{Fee-for-Service Subsample Analysis}

This analysis utilized a claims-based measure of nursing home admission rather than residency, which identified an admission in the 60 days prior to hospice use for $28 \%$ of FFS hospice users (compared to only 3.3\% nursing home residents identified by AFU telephone interview, usually within a year prior to hospice initiation). Estimated ORs (Table 4) for the effect of being in a nursing home were not significant in any of the models, possibly due to the smaller sample size $(\mathrm{N}=791)$. However, persons having a nursing home admission within 60 days prior to hospice were more likely to have short stays and less likely to be discharged alive, which was different than the effect of nursing home residency in the full sample analysis. For the other variables, the estimated associations in the FFS analysis were similar to the full sample.

\section{Discussion}

Trends in hospital use over the year prior to hospice initiation are associated with hospice length of stay and live discharge. The odds of a short hospice stay were significantly higher for hospice patients who experienced increasing hospital use. Increasing hospital use was also associated with reduced odds of a long stay in hospice or a live discharge from hospice. These results were robust in the FFS subsample analysis using different measures of comorbidity and nursing home status. Associations of other factors (hospice qualifying diagnosis, nursing home status, marital status, age, and community residence) with hospice episode characteristics were generally consistent with existing studies; however, we failed tofind evidence that the trends in hospital use in the year prior to hospice initiation are different for different diagnosis groups conditional on having a hospital admission in the study period. This null result warrants further study potentially using alternative definitions of care intensity and incorporating outpatient and emergency department use.

The association of hospital use with hospice outcomes may inform policies or practices aiming to improve the timing of hospice initiation. Prior research has shown utilization of high-intensity services in the days prior to hospice admission is more likely for users with short hospice episodes, and our results suggest that this pattern of high-intensity care among those with short hospice stays may develop over the year preceding hospice use." Currently, Medicare beneficiaries are required to forgo curative care in order to enroll in hospice. As a result, the association of increasing time in the hospital with a short hospice stay may occur as patients, caregivers, and clinicians elect to try all available treatments before defining a patient as terminally ill and subsequently choosing hospice care when death is imminent. 
Other beneficiaries may be relatively healthy initially but experience a sharp decline in health that results in hospitalization and transfer to hospice, with death following shortly after. Uncertainty among clinicians regarding prognosis and life expectancy may also contribute to short hospice stays.

Although a short stay in hospice is not always an undesirable or preventable outcome, our study suggests that trends in hospital use may be useful for interventions improving the timing of hospice for those with a stay that is too short to meet the needs of the patient. The CMS is currently implementing the Medicare Care Choices Model, a demonstration project, which would allow hospice-like support services as a concurrent care strategy for patients who wish to continue curative therapy. Given this option, patients who are experiencing an increasing need for care may wish to receive this limited package of hospice support services earlier in the trajectory of care. Increasing hospital use in Medicare patients may be an indicator that a conversation about end-of-life care is warranted, if not begun already. These trends may be used to identify subsets of potential hospice users earlier in the trajectory of care, providing better quality of life and patient and caregiver satisfaction.

There is also a concern that hospice is initiated too early in the care trajectory, resulting in a live discharge. Among Medicare FFS hospice beneficiaries, about $18 \%$ of discharges are live and 1 in 4 of those discharged alive are hospitalized within 30 days. The analysis in this article suggests that initiating discussions with patients with increasing intensity of care has the potential to improve the timing of hospice while reducing the proportion of live discharges in the population of hospice users.

\section{Limitations}

The ARIC sample of Medicare enrollees from 4 communities is not nationally representative. For example, only $13 \%$ of ARIC hospice users were older than 85 years during the study period, compared to about $40 \%$ of hospice users in the United States. As with most hospice analyses, potentially relevant factors like caregiver availability or characteristics were not available. Furthermore, this analysis did not consider the care patterns of those who did not use hospice, which precludes the evaluation of causal relationships with hospice initiation. Despite these concerns, ARIC data provide individual characteristics not available via traditional sources of claims data including education and marital status as well as more complete hospitalization information. The inclusion of these variables reduces potential bias due to omitted variables.

\section{Conclusion}

This study provides novel information about trends in hospital use over the year prior to hospice initiation. The results suggest that prehospice patterns of care, specifically increasing intensity of inpatient services, may be a useful indicator for clinicians to identify patients and caregivers for whom a discussion about patient and family goals for care may be appropriate. Further study of prehospice patterns of care may inform the development of policies that will improve the timing of hospice enrollment and maximize the benefits of hospice care. 


\section{Acknowledgments}

The authors thank the staff and participants of the ARIC study for their important contributions.

Funding

The author(s) disclosed receipt of the following financial support for the research, authorship, and/or publication of this article: The ARIC study is carried out as a collaborative study supported by National Heart, Lung, and Blood Institute contracts (HHSN268201100005C, HHSN268201100006C, HHSN268201100007C, HHSN2682011000 08C, HHSN268201100009C, HHSN268201100010C, HHSN2682011 00011C, and HHSN268201100012C).

\section{References}

1. Agar M, Currow DC, Shelby-James TM, Plummer J, Sanderson C, Abernethy AP. Preference for place of care and place of death in palliative care: are these different questions? Palliat Med. 2008;22(7):787-795. [PubMed: 18755830]

2. Waldrop DP, Meeker MA. Final decisions: how hospice enrollment prompts meaningful choices about life closure. Palliat Support Care. 2014;12(3):211-221. [PubMed: 23942112]

3. Teno JM, Clarridge BR, Casey V, et al. Family perspectives on end-of-life care at the last place of care. JAMA. 2004;291(1): 88-93. [PubMed: 14709580]

4. Patrick DL, Engelberg RA, Curtis JR. Evaluating the quality of dying and death. J Pain Symptom M anage. 2001;22(3): 717-726.

5. Lorenz KA, Asch SM, Rosenfeld KE, Liu H, Ettner SL. Hospice admission practices: where does hospice fit in the continuum of care? J Am Geriatr Soc. 2004;52(5):725-730. [PubMed: 15086652]

6. Teno JM, Gozalo PL, Bynum JP, et al. Change in end-of-life care for Medicare beneficiaries: site of death, place of care, and health care transitions in 2000, 2005, and 2009. JAMA. 2013;309(5): 470477. [PubMed: 23385273]

7. Facts and Figures on Hospice Care. 2012; Web site. http://www.nhpco.org/sites/default/files/public/ Statistics_Research/2011_Facts_Figures.pdf. Accessed July 3, 2016.

8. Yamagishi A, Morita T, Kawagoe S, et al. Length of home hospice care, family-perceived timing of referrals, perceived quality of care, and quality of death and dying in terminally ill cancer patients who died at home. Support Care Cancer. 2015;23(2): 491-499. [PubMed: 25142705]

9. Waldrop DP, Rinfrette ES. Can short hospice enrollment be long enough? Comparing the perspectives ofhospice professionals and family caregivers. Palliat Support Care. 2009;7(1):37-47. [PubMed: 19619373]

10. Teno JM, Shu JE, Casarett D, Spence C, Rhodes R, Connor S. Timing of referral to hospice and quality of care: length of stay and bereaved family members' perceptions of the timing of hospice referral. J Pain Symptom Manage. 2007;34(2):120-125. [PubMed: 17583469]

11. Johnson KS, Kuchibhatla M, Tulsky JA. Racial differences in location before hospice enrollment and association with hospice length of stay. J Am Geriatr Soc. 2011;59(4):732-737. [PubMed: 21410443]

12. Cheung WY, Schaefer K, May CW, et al. Enrollment and events of hospice patients with heart failure vs. cancer. J Pain Symptom Manage. 2013;45(3):552-560. [PubMed: 22940560]

13. Lawrence VA, Hazuda HP, Cornell JE, et al. Functional independence after major abdominal surgery in the elderly. J Am Coll Surg. 2004;199(5):762-772. [PubMed: 15501119]

14. Gadoud A, Jenkins SM, Hogg KJ. Palliative care for people with heart failure: summary of current evidence and future direction. Palliat Med. 2013;27(9):822-828. [PubMed: 23838375]

15. Brickner L, Scannell K, Marquet S, Ackerson L. Barriers to hospice care and referrals: survey of physicians' knowledge, attitudes, and perceptions in a health maintenance organization. J Palliat Med. 2004;7(3):411-418. [PubMed: 15265350]

16. Kennedy C, Brooks-Young P, Brunton Gray C, et al. Diagnosing dying: an integrative literature review. BM JSupport Palliat Care. BM J Support Palliat Care. 2014;4(3):263-270.

17. Unroe KT, Sachs GA, Hickman SE, Stump TE, Tu W, Callahan CM. Hospice use among nursing home patients. J Am M ed Dir Assoc. 2013;14(4):254-259. 
18. Miller SC, Lima JC, Mitchell SL. Hospice care for persons with dementia: the growth of access in US nursing homes. Am J Alzheimers D is Other Demen. 2010;25(8):666-673.

19. Kompanje EJ, Piers RD, Benoit DD. Causes and consequences of disproportionate care in intensive care medicine. Curr Opin Crit Care. 2013;19(6):630-635. [PubMed: 24240830]

20. Barlow AP, Zarifa Z, Shillito RG, Crumplin MK, Edwards E, McCarthy JM. Surgery in a geriatric population. Ann R Coll Surg Engl. 1989;71(2):110-114. [PubMed: 2705717]

21. Taylor DH, Jr, Ostermann J, Van Houtven CH, Tulsky JA, Stein-hauser K. What length of hospice use maximizes reduction in medical expenditures near death in the US Medicare program? Soc Sci Med. 2007;65(7):1466-1478. [PubMed: 17600605]

22. Aaltonen M, Raitanen J, Forma L, Pulkki J, Rissanen P, Jylha M. Burdensome transitions at the end of life among long-term care residents with dementia. J Am M ed D ir Assoc. 2014;15(9): 643-648.

23. Baldwin MR, Narain WR, Wunsch $\mathrm{H}$, et al. A prognostic model for 6-month mortality in elderly survivors of critical illness. Chest. 2013;143(4):910-919. [PubMed: 23632902]

24. Creditor MC. Hazards of hospitalization of the elderly. Ann Intern Med. 1993;118(3):219-223. [PubMed: 8417639]

25. The ARIC Investigators. The Atherosclerosis Risk in Communities (ARIC) study-design and objectives. Am J Epidemiol. 1989;129(4):687-702. [PubMed: 2646917]

26. The Atherosclerosis Risk in Communities (ARIC) Study: design and objectives. The ARIC investigators. Am J Epidemiol. 1989; 129(4):687-702. [PubMed: 2646917]

27. Miller SC, Kinzbrunner B, Pettit P, Williams JR. How does the timing of hospice referral influence hospice care in the last days of life? J Am Geriatr Soc. 2003;51(6):798-806. [PubMed: 12757566]

28. Farnon C, Hofmann M. Factors contributing to late hospice admission and proposals for change. Am J Hosp Palliat Care. 1997;14(5):212-218. [PubMed: 9341336]

29. Chung K, Essex E, Samson LF. Ethnic variation in timing of hospice referral: does having no informal caregiver matter? J Palliat Med. 2008;11(3):484-491. [PubMed: 18363492]

30. Chung K, Essex EL, Samson L. Does caregiver knowledge matter for hospice enrollment and beyond? Pilot study of minority hospice patients. Am J H osp P alliat Care. 2009; 26(3):165-171.

31. Kirkendall A, Shen JJ, Gan Y. Associations of race and other socioeconomic factors with posthospitalization hospice care settings. Ethn Dis. 2014;24(2):236-242. [PubMed: 24804373]

32. Hardy D, Chan W, Liu CC, et al. Racial disparities in the use of hospice services according to geographic residence and socioeconomic status in an elderly cohort with nonsmall cell lung cancer. Cancer. 2011;117(7):1506-1515. [PubMed: 21425152]

33. Heathcare Cost and Utilization Project Co-Morbidity Software Version 3.7 Web site. http:// www.hcup-us.ahrq.gov/toolssoftware/comorbidity/comorbidity.jsp. Accessed July 3, 2016.

34. Charlson ME, Pompei P, A les KL, M acKenzie CR. A new method of classifying prognostic comorbidity in longitudinal studies: development and validation. J Chronic D is. 1987; 40(5):373383.

35. Huang Y, Gou R, Diao Y, et al. Charlson comorbidity index helps predict the risk of $\mathrm{m}$ ortality for patients w ith type 2 diabetic nephropathy. J Zhejiang Univ Sci B. 2014;15(1): 58-66. [PubMed: 24390745]

36. CMS Chronic Condition Data Warehouse Condition Categories. 2010; Web site. https:// www.ccwdata.org/web/guest/condition-categories. Accessed July 3, 2016.

37. Zuckerman IH, Sato M, Hsu VD, Hernandez JJ. Validation of method for identifying nursing home admissions using administrative claims. BM C Health Serv Res. 2007;7(1):202.

38. Centers for Medicaid and Medicare Services. Medicare Choices Model Awards. Fact Sheets 2015; Web site. http://www.cms.govNewsroom/MediaReleaseDatabase/Fact-sheets/2015-Fact-sheetsitems/2015-07-20.html. Accessed July 24, 2015.

39. Teno JM, Plotzke M, Gozalo P, Mor V. A national study of live discharges from hospice. J P a llia t M ed. 2014;17(10): 1121-1127.

40. Organization NHaPC. Facts and Figures on Hospice Care. 2012; Web site. http://www.nhpco.org/ sites/default/files/public/Statistics_Research/2011_Facts_Figures.pdf. Accessed April 13, 2015. 
41. Mood C Logistic regression: why we cannot do what we think we can do, and what we can do about it. Eur Soc Rev. 2010;26(1): 67-82. 


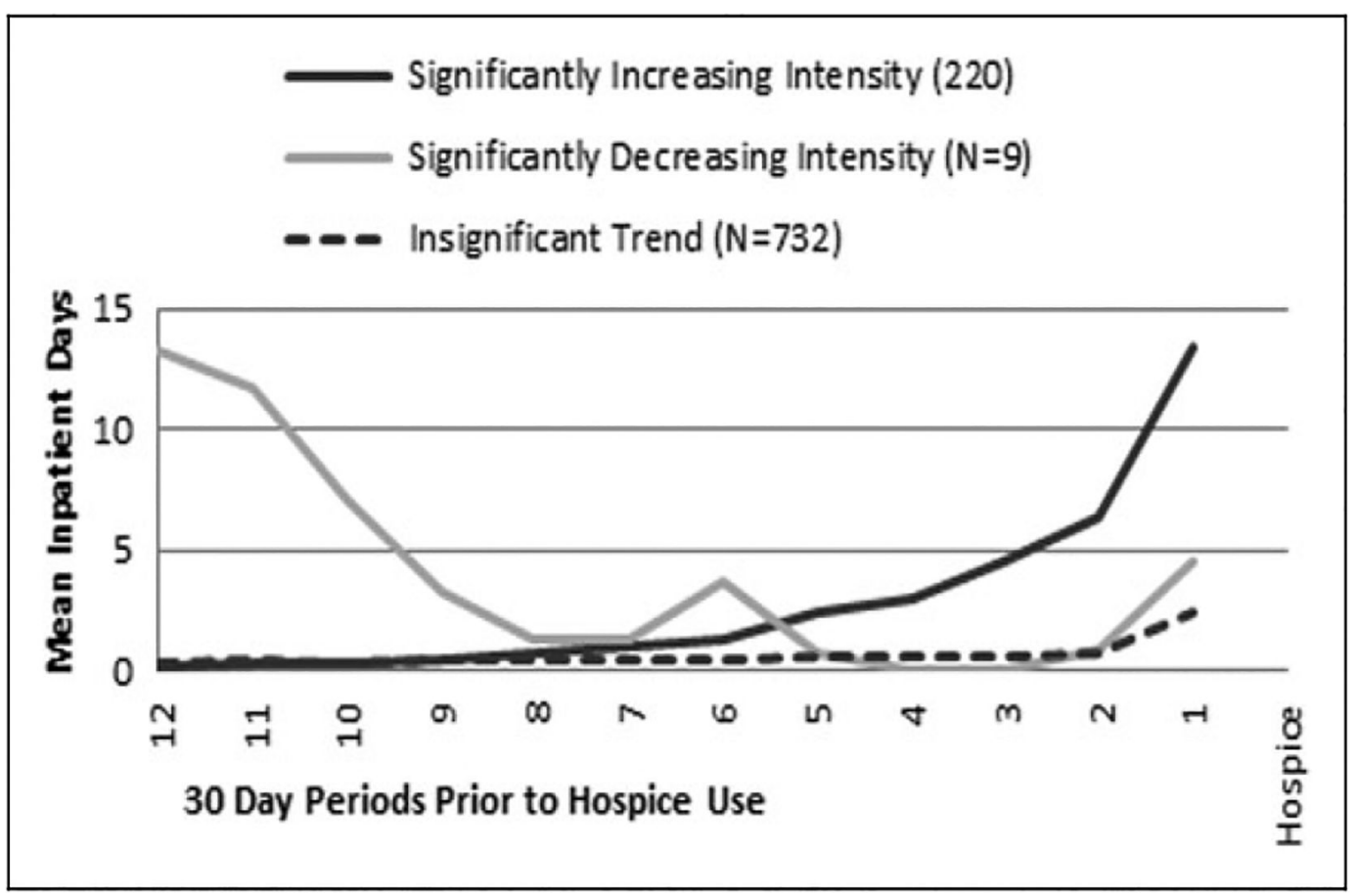

Figure 1.

Trends in hospital use over the year prior to hospice admission by pattern of care (961 with at least 1 hospitalization during the year prior to hospice enrollment). Participants with no hospital days in the time period are excluded. Those with hospital days are grouped by trend in hospital use. Not all participants have a hospitalization in each 30-day period, so the average number of days per user per period is less than the average length of hospital stay in sample (6 days). 


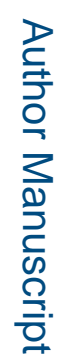

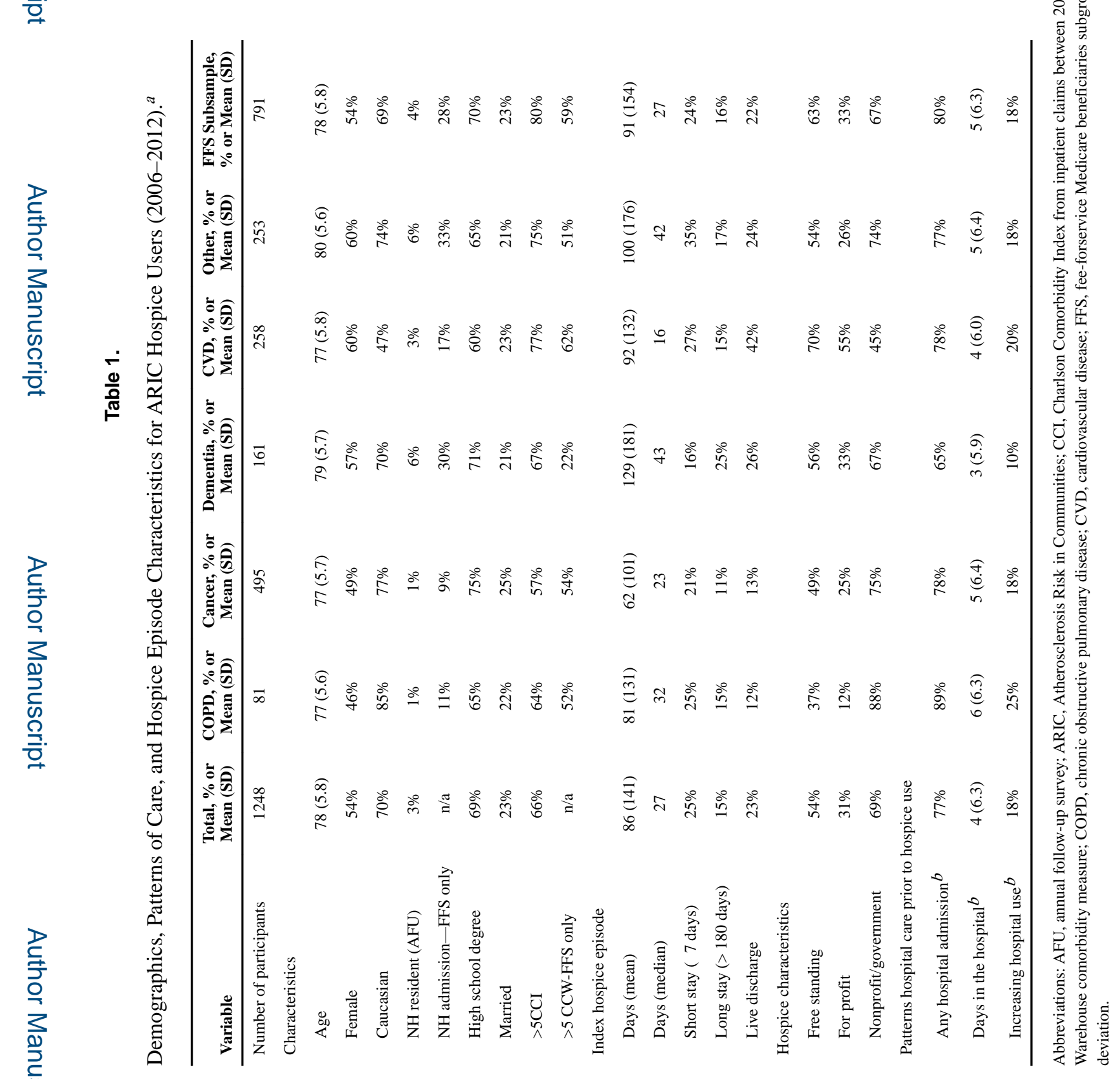

Am J Hosp Palliat Care. Author manuscript; available in PMC 2018 October 09. 


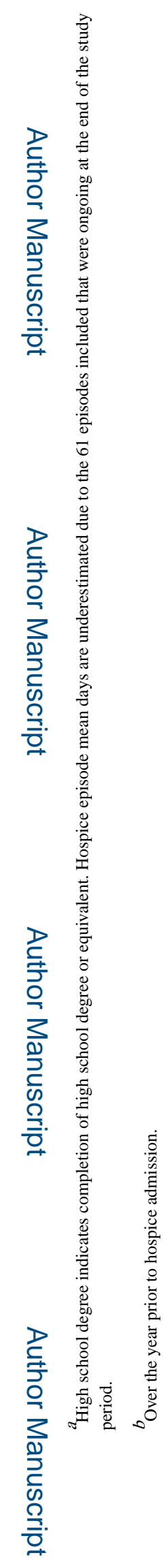

Am J Hosp Palliat Care. Author manuscript; available in PMC 2018 October 09. 
(a)

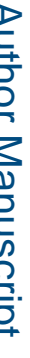

\section{}

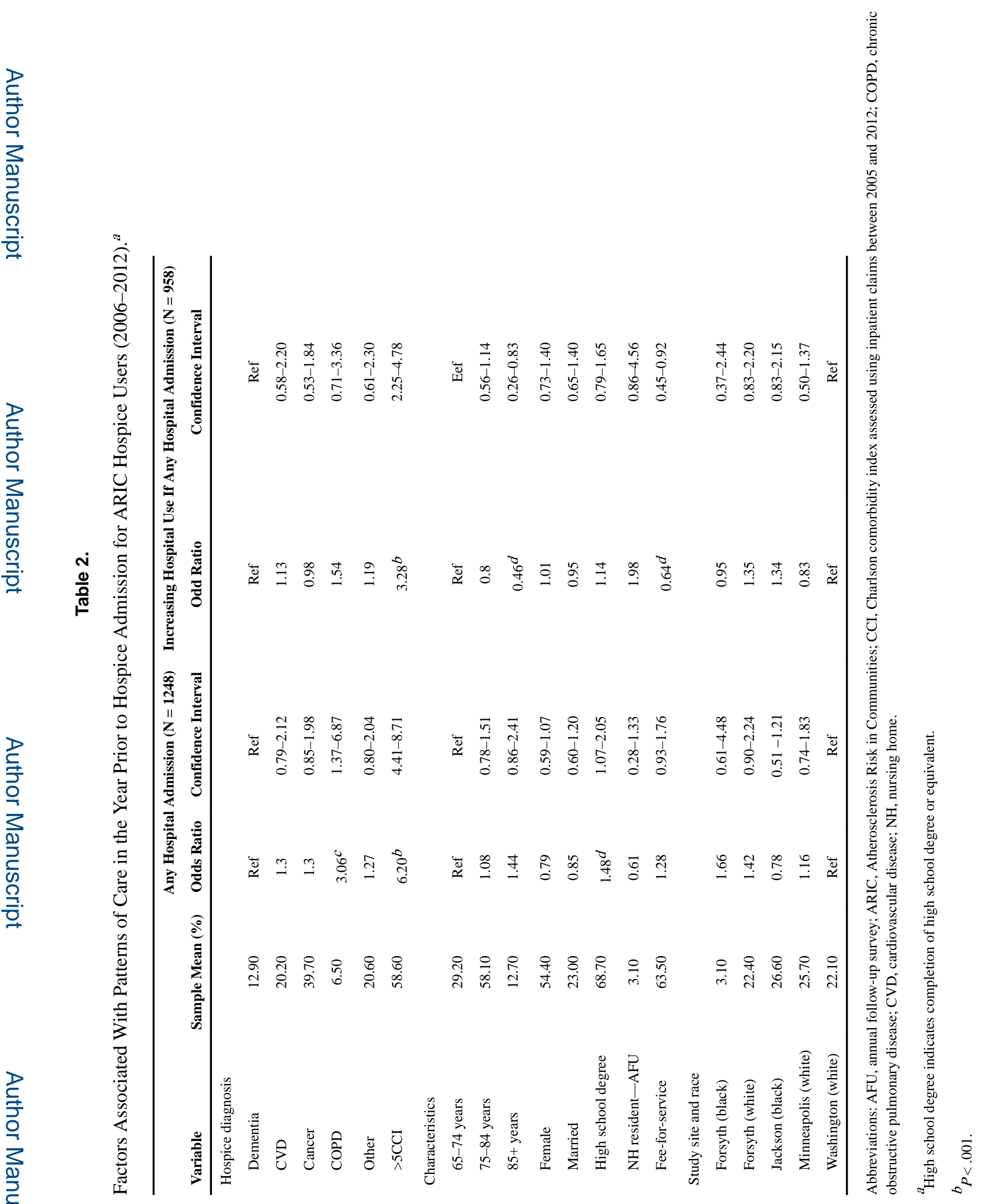

Am J Hosp Palliat Care. Author manuscript; available in PMC 2018 October 09. 


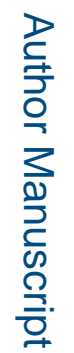




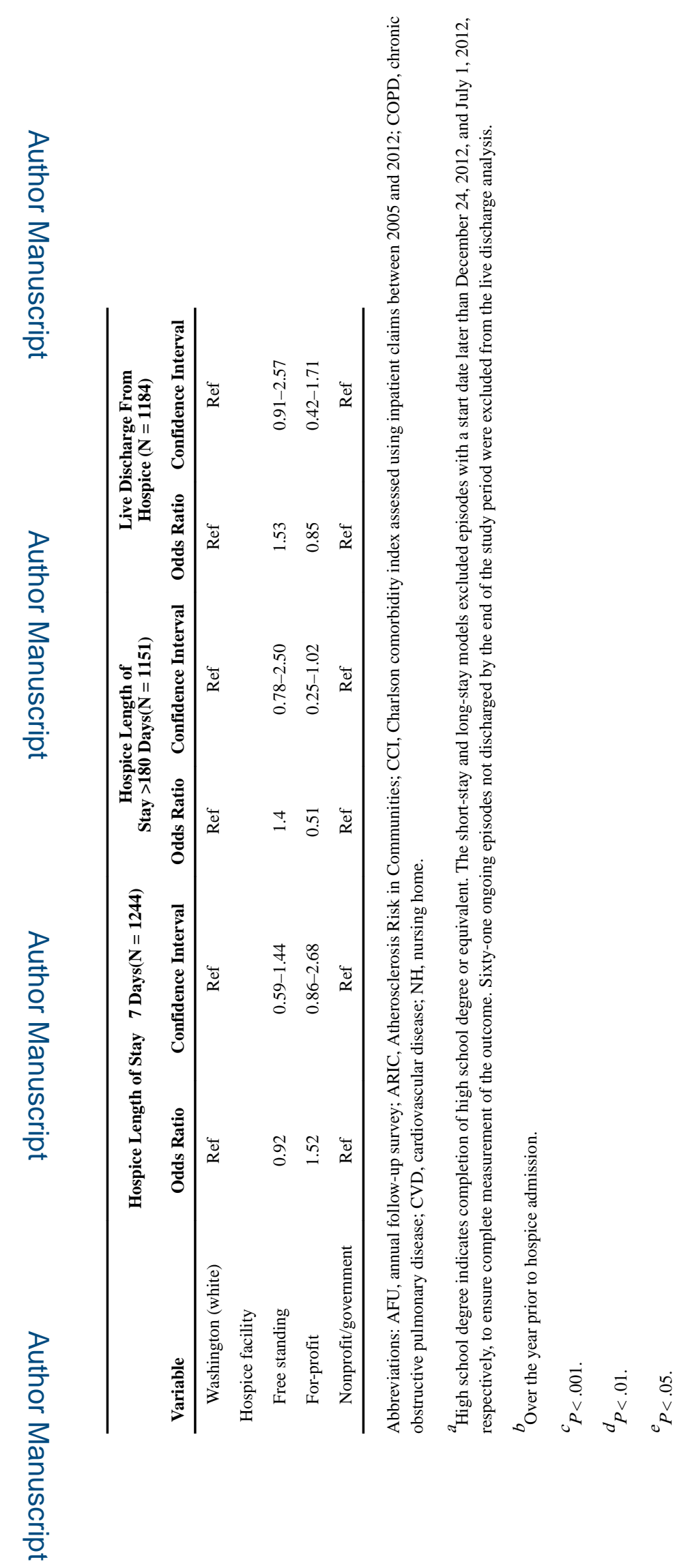

Am J Hosp Palliat Care. Author manuscript; available in PMC 2018 October 09. 


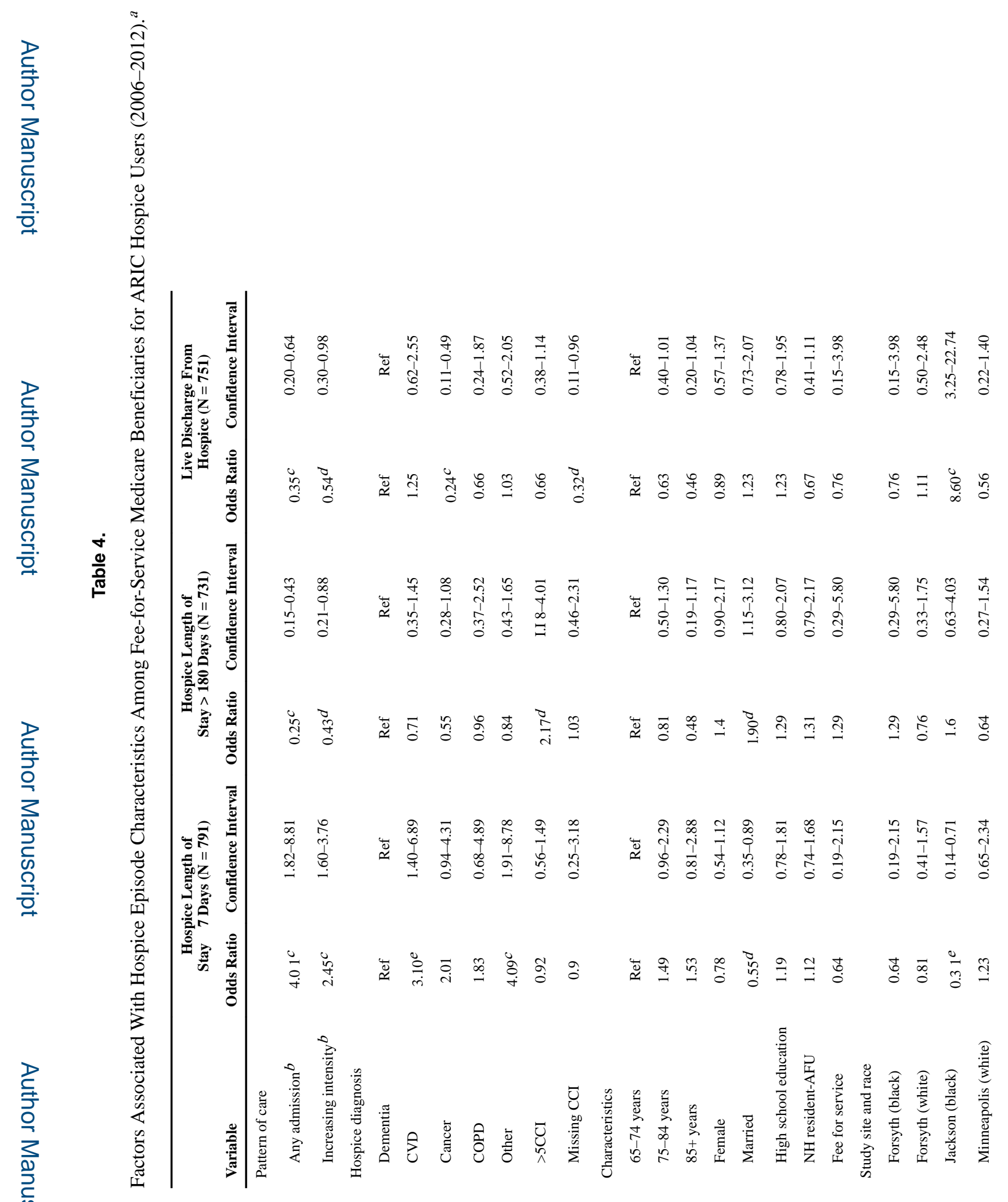

Am J Hosp Palliat Care. Author manuscript; available in PMC 2018 October 09. 


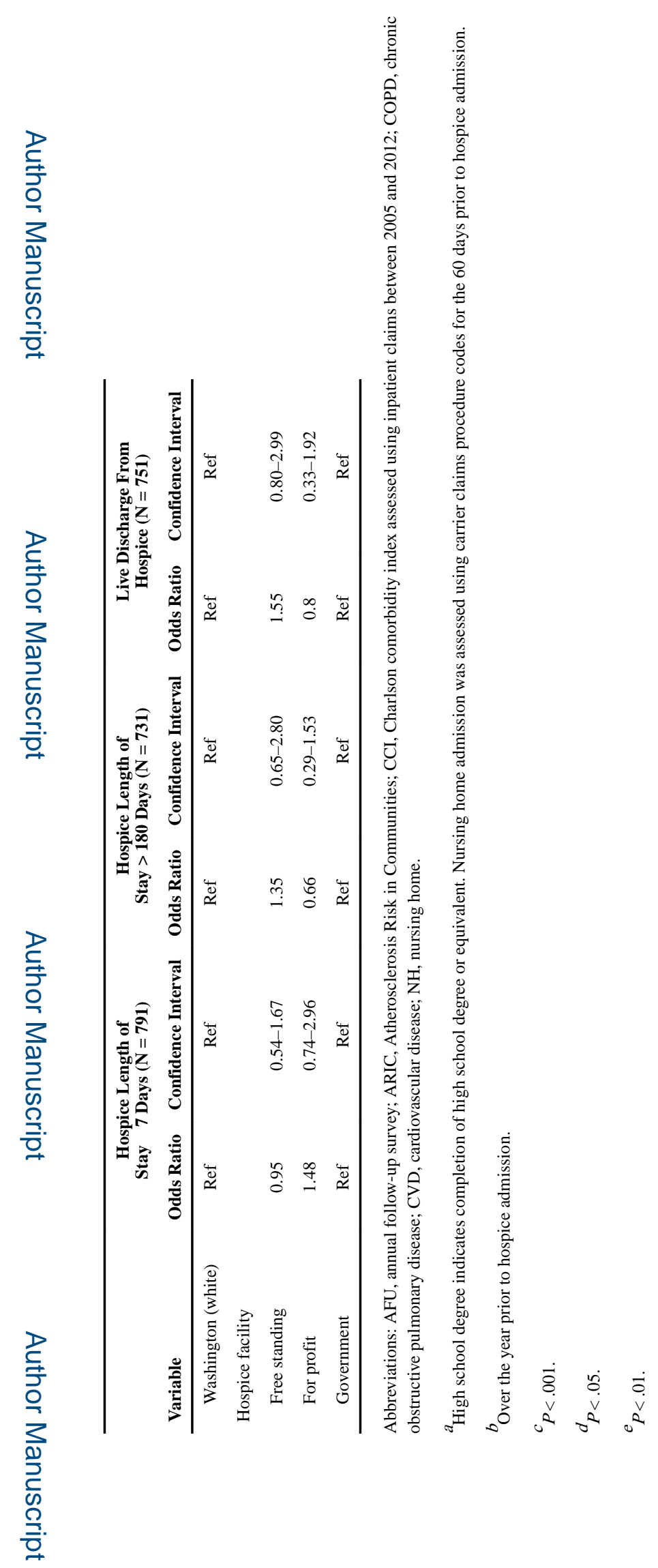

Am J Hosp Palliat Care. Author manuscript; available in PMC 2018 October 09. 